\title{
Assessing Maize Foliar Water Stress Levels Under Field Conditions Using In-Situ Spectroscopy
}

\author{
A. Ngie $^{1 *} ;$ F. Ahmed ${ }^{1} ;$ K. Abutaleb ${ }^{1}$ \\ ${ }^{1}$ University of the Witwatersrand, Johannesburg, South Africa \\ *Corresponding author: email: adelinengie@gmail.com
}

Abstract: Plant physiological processes required for crop productivity are dependent on the availability of water to crops. Water availability to crops therefore requires real time monitoring for timeous rescue or intervention measures. Such monitoring over vast areas is only possible through remotely sensed techniques such as spectroscopy with its numerous fine wavelengths and is non-destructive to the crops as opposed to other traditional ground-based methods. The management of spectral reflectance data to extract information of importance for plant water status has been motivated by knowledge of the availability of specific bands in the electromagnetic spectrum responsible for water absorption. The purpose of this study was to investigate the potential of using selected spectral bands to develop water indices that could monitor the water status at leaf level on maize (Zea mays) plants grown under field conditions. Leaf spectral reflectance of maize plants was collected under three different water conditions being healthy $(\mathrm{H})$, intermediary water stressed (IWS) and water stressed (WS) using a leaf-clip of a handheld spectroradiometer. The spectral reflectance indicated an increased reflectance in portions of the visible, near-infrared and short infrared regions of the electromagnetic spectrum for the water stressed maize plants. The random forest (RF) algorithm was utilised to extract wavelengths of importance from which water indices were developed among which were the normalised difference water index (NDWI860-1240) and the water band index (WBI950-970). The indices were used in a combined algorithm of RF and partial least square (PLS) for its predictive ability to classify the maize leaf water status into the three categories (H, IWS and WS). The results showed an overall accuracy of $70 \pm 1.2 \%$. Therefore, confirming the potential of assessing leaf water content using in-situ spectroscopy. The three most important indices were NDWI $860-1240, \mathrm{NDWI}_{1700-1530}$ and NDWI 1530-1360. An in-depth study would be required to quantify and measure actual water content in maize leaves and possibly upscale to canopy level which would directly support irrigation management plans.

Keywords: in-situ spectroscopy, maize, partial least square, random forest, water spectral indices, water stress 


\section{INTRODUCTION}

\section{A. Background on plant water availability}

Water is a key determinant in field crop productivity globally though its availability is highly variable. Challenging climate scenarios of unprecedented spatio-temporal temperature and rainfall patterns does translate into changes in availability of water to crops (IPCC, 2007). Accurate water content estimation is required to make decisions on management schemes and also crop yield estimation in agricultural studies (Peñuelas et al., 1993). The water content/status of a plant can be measured from root, stem and leaf material or the whole canopy. Leaf analyses are however, the most important for evaluating the nutrient and water status of plants in comparison to other tissue types (Suo et al., 2010).

Leaf water content has been considered as an indirect estimate of the changes in the water status in the leaves and could be measured through the reactions of altered plant cell structures (Canny \& Huang, 2006). The leaf is also mostly responsible for photosynthesis, an essential physiological process in plants. The health and nutrient status with water status inclusive of the plants can be evaluated from the leaves where its decrease would serve as an important indicator of stress or a limiting factor (de Jong et al., 2012). Therefore, leaf water content can be used for the determination of the water status of the plants. Initially, plant water stress has been measured through destructive approaches that are limited in spatial extent as a result of being labour intensive (Graeff \& Claupein, 2007).

The basis of detecting water stress with remote sensing relates to the difference in reflectance properties of plants under different water stress levels at certain wavelengths in the NIR portion of the electromagnetic spectrum (Genc et al., 2013). The interpretation of these hyperspectral data is complicated by the interrelationships between wavelength variables which require extensive statistical techniques to analyse the data for meaningful information to be derived. While some studies made use of the leaf spectral reflectance directly to assess leaf water content (Gaussman, 1977; Tucker, 1980; Hunt \& Rock, 1989; Marraci et al., 1991), others have perform data transformations and developed water indices (Ceccato et al., 2002; Sims \& Gamon, 2003; Govender et al., 2009) to aid in the generation of information about the water status in plants.

\section{B. Leaf water content and water indices}

Water indices have been developed to evaluate plant water content at both leaf and canopy levels over the years the most frequently used ones include the water band index (WBI) (Peñuelas et al., 1993), normalized difference water index (NDWI) (Gao, 1995; Serrano et al., 2000) and recently the shortwave infrared water stress index (SIWSI) (Fensholt \& Sandholt, 2003). These indices were developed from the combinations of bands or wavelengths from the NIR (WBI and NDWI) and the SWIR (SIWSI). However, more recently there have been 
attempts on using the blue, green, red wavelengths or bands (VIS region) and the NIR wavelengths in developing indices to estimate vegetation water content (Genc et al., 2013).

The bands identified as suitable for water status assessment would not be exactly the same for all crops but serve as a guide to band centres for water absorption (Danson et al., 1992; Penuelas et al., 1993; Sims \& Gamon, 2003; Clevers et al., 2008 \& 2010; Elsayed et al., 2011; Genc et al., 2013). The minor water-absorption bands which are situated around 970 and $1200 \mathrm{~nm}$ have proven ability to provide and or even quantify leaf water status in plants more than the major bands of the 1400 and $1900 \mathrm{~nm}$ region of the electromagnetic spectrum (Clevers $e t$ $a l ., 2010)$. The spectral indices developed from the minor water absorption band centres have also proven feasible (correlation of 0.70 on the right slope of $970 \mathrm{~nm}$ ) in determining foliar water status (de Jong et al., 2014).

The water band index (WBI) is derived from the ratio of reflectance measured at 950 and $970 \mathrm{~nm}$ (Peñuelaset al., 1993); and 900 - $970 \mathrm{~nm}$ (Peñuelaset al., 1997). This spectral index has been correlated with ground-based measurements of plant water content at both the leaf and canopy scales. It is, however, more sensitive to leaf water content than the water content of the whole plant. This is advantageous in agricultural applications where leaf water content changes more noticeably in response to drought conditions than the water content of the entire plant foliage (Champagne et al., 2003).

The normalised difference water index (NDWI) has been another widely used index for monitoring water status of vegetation both through multispectral (Jackson et al., 2004) and hyperspectral (Eitel et al., 2006; Elsayed et al., 2011; Winterhalter et al., 2011) remote sensing. It is measured by a ratio of difference between the reflectance value at 860 and $1240 \mathrm{~nm}$ wavelengths (Gao, 1996). The index is measured of wavelengths both from the NIR region of the electromagnetic spectrum and its application in detecting water content has been widely used (Govender et al., 2009). However, other wavelengths have been used to develop ratios which have also illustrated important as a result of crop types or management conditions in monitoring water status (Winterhalter et al., 2011).

The SWIR (1400-2500) has also proven important in leaf water content measurement with the identification of the 1550 and $1750 \mathrm{~nm}$ wavelengths (Tucker, 1980); and in recent studies the VIS region was also identified as important in water status assessment (Graeff \& Claupein, 2007; Genc et al., 2013). The detection of leaf water content in the VIS/NIR regions linking it to chlorophyll and nitrogen status was recently assessed with successful outcomes (Zhang et al., 2012) whereas others previously realized only the NIR and SWIR regions to be important in leaf water content assessment (Ceccato et al., 2001).

Hyperspectral remote sensing therefore, is able to provide information on rapidly occurring water status changes in plants driven by dynamism in immediate environmental conditions. Maize (Zea mays) being an important field 
crop that relies on water availability for enhanced productivity has been tested in monitoring with this technology (Elsayed et al., 2011; Winterhalter et al., 2011; Genc et al., 2013). It is clear that water status assessment varies across crop types and geographical locations as a result of varying water budgets. What is clearly more important is that water stress affects crop productivity and thus requires monitoring.

This study then seeks to utilise known spectral indices such as NDWI860/1240 and WBI950/970 together with others developed from selected wavelengths in detecting water status to distinguish different water status in maize leaves under field conditions. Firstly, the spectral data is resampled to $10 \mathrm{~nm}$ and a feature selection is performed to extract wavelengths of importance that contribute to the detection of water status in the maize leaves using in-situ

spectral measurements. Secondly, the selected wavelengths were used to develop spectral indices. Lastly, the indices are all used as independent variables for the classification of the various water status of the maize leaves while ranking them according to importance based on the error size obtained when running the permutations without each index.

\section{MATERIALS AND METHODS}

\section{Study area}

The fields used for this study were located at the Glen Agricultural College fields in Bloemfontein (Free State province of South Africa) with geographical coordinates as $28^{\circ} 56^{\prime} 45.86^{\prime \prime} \mathrm{S}$, 26²'19'35.93" E (maize field) and 28 56'53.34" S, 26²19'41.49" E (adjacent field). The fields were located about $20 \mathrm{~km}$ north of Bloemfontein (Figure 2). The area has mean annual precipitation of about $600 \mathrm{~mm}$ and mean annual maximum and minimum temperatures of $25^{\circ} \mathrm{C}$ and $8^{\circ} \mathrm{C}$ respectively (Botha et al., 2007). Summers are hot and dry with scarce episodes of rainfall while the winters are frosty and cold. The growing of crops such as maize is only done once in a summer farming season that commences in December and runs through to July. As a result of its sparse rainfall, the growth of crops is through irrigation schemes.

\section{Field design}

The field used for this study was grown under the same conditions with the same maize cultivar (PAN 6616) on the $15^{\text {th }}$ of January 2014. It was grown under irrigation scheme but the amount of water applied could not be measured since it was the flooding system. This flooding system meant the whole field does not receive water equally, thereby creating variable water availabilities to the plants across the field. The area located close to the water source gets saturated with water before it flows to the other parts of the field. Hence, the areas furthest from the water source stay water stressed 
and in between the two areas is an intermediary scenario with limited amounts of water available to the plants (Figure 1).

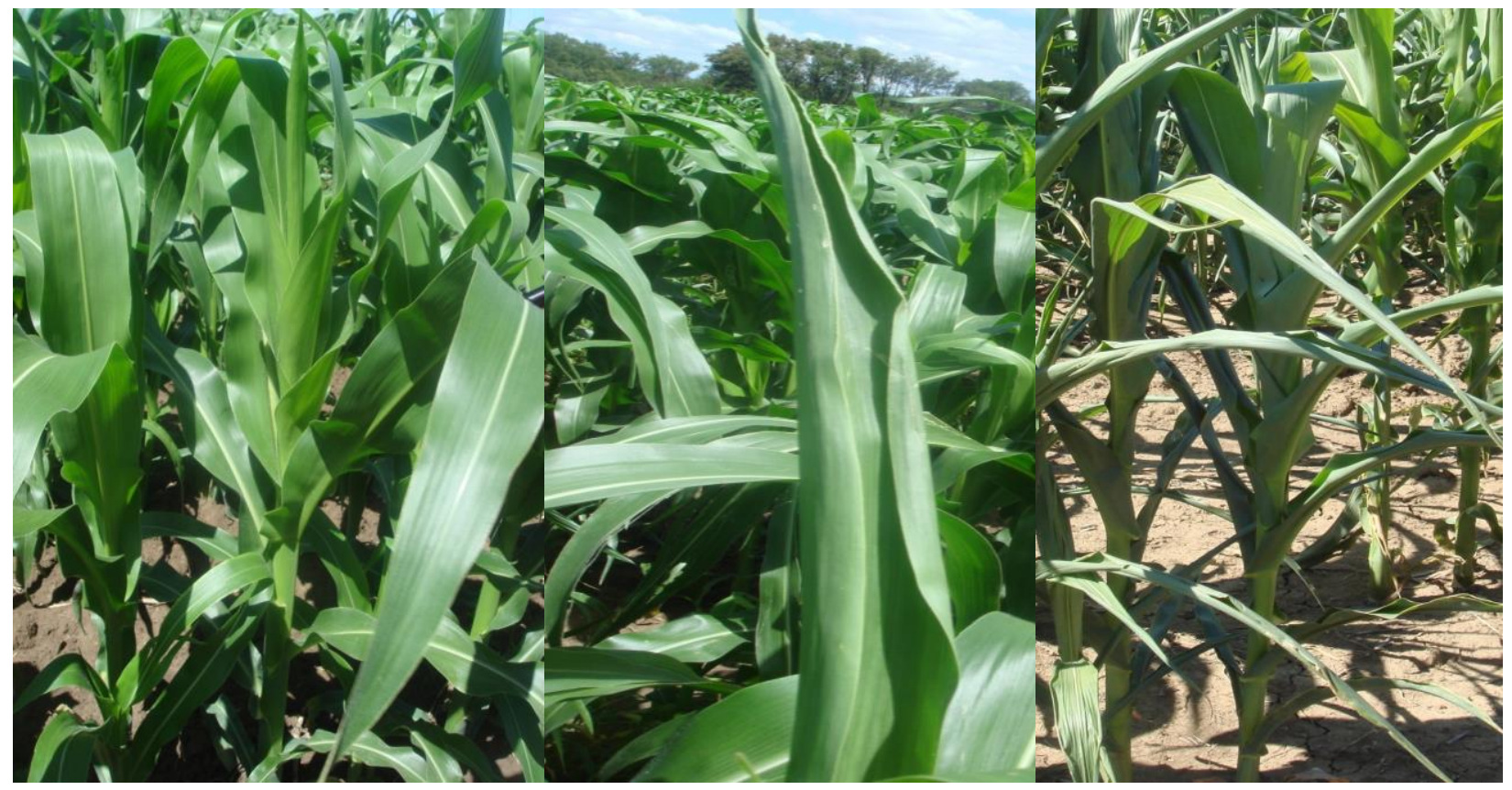

Figure 1: Maize leaves showing the different categories of water status on the day of spectral measurements: $\mathbf{a}$ is healthy or unstressed leaves; $\mathbf{b}$ is intermediary water stressed leaves; and $\mathbf{c}$ is water stressed leaves 


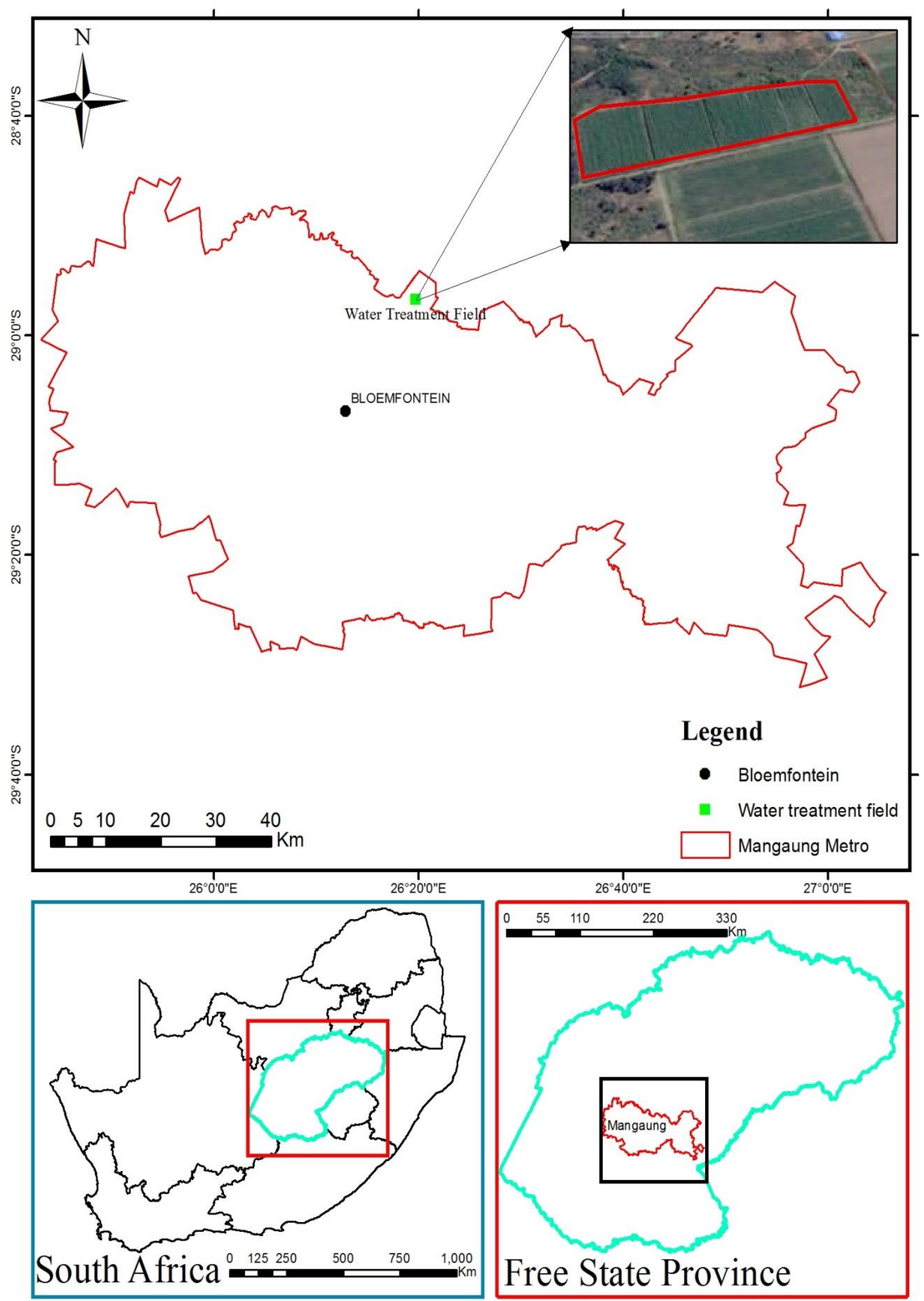

Figure 2: Study area showing the maize field in Glen, Bloemfontein. (Insert image from GoogleEarth $4 / 10 / 2014$ 


\section{E. Spectral measurement and pre-processing}

The spectra were collected on the $11^{\text {th }}$ of April 2014 (11-12 weeks after planting) by selecting the top-most completely opened leaf. The measurements were taken in the morning period between 10 am and 12 noon to avoid the interference from the process of evapotranspiration and the difference in water potential between the plants are at their greatest (Cleary \& Zaerr, 1984). The spectral measurements were done on the adaxial surface of each leaf using the leaf-clip device of the handheld portable PSR-3500 spectroradiometer (@2012 Spectral Evolution, Inc, USA), with the average scan time set at $10 \mathrm{~ms}$ (averaged to reduce scanner noise). The leaf-clip was used in this case as a result of its direct-contact probe which limits ambient light.

The spectroradiometer system operates in the range of 340-2500 $\mathrm{nm}$. The complete system collects and stores data which is calibrated to units of spectral radiance (W/m²/nm/sr) as output (PSR, 2012). The non-destructive measurements in the field were obtained on both sides of the midrib but avoiding the midrib region. The radiance measurements were calibrated using a white spectralon reference panel before scanning the leaves and converted to relative reflectance $(\%)$.

The spectra were measured at a total of 1024 wavelengths with a spectral range of 0 to $4 \mathrm{~nm}$. In order to reduce the number of wavelengths, the spectral measurements were resampled using a Gaussian model (full-width half-maximum) in the Environment for Visualizing Images (ENVI) software (v.4.5, ITT Visual Information Systems) that resulted in 217 wavelengths with a $10 \mathrm{~nm}$ spectral range.

\section{Data analysis}

\section{F. Wavelength selection and development of indices}

Data analysis was performed in RStudio using the random forest (RF) algorithm. RF is an ensemble approach that is capable of performing a divide-and-conquer rule that improves performance of data management (Brieman, 2001). This is done through recursive partitioning of the data into subsets (trees) known as ntree drawing random subsets of variables known as mtry (ibid). The RF is a machine learning algorithm that has the potential of handling huge data sets of large predictor variable numbers (wavelengths) as oppose to the small sample size. In order to assess the impact of a predictor variable, the RF permutation of importance measured is biased due to the preferential selection of correlated 
wavelengths (Stroblet al., 2007). The bias is adjusted in the conditional random forest (cforest) proposed by Strobl et al. (2008).

Every tree is constructed using a different bootstrap sample from the original data that gets partitioned into smaller subsets or trees that are combined with the notion of an ensemble at the nodes. The RF algorithm uses two thirds ( $2 / 3$ or $70 \%$; 13 samples) of the data (training data set) in every bootstrap sample while a third (1/3 or $30 \%$; 7 samples) being the test data set is left out of the construction and used for validation where an error estimate is generated for every predictive variable. The error estimate is known as the out-of-bag (OOB) error and measures the variable of importance in the algorithm. For cforest the $n$ tree were tested by 50 s up to 500 and the $m$ try as $\sqrt{n}$ where $\mathrm{n}$ is the 217 wavelengths. Hence the mtry was 15.

The top 10 wavelengths in the entire spectrum as ranked by the OOB errors were selected and used to develop the spectral vegetation indices relating to the NDWI (Equation 1). These wavelengths were considered as those of most informative to water status in maize leaves under field conditions.

$$
N D W I=\frac{\lambda_{N I R}-\lambda_{S W I R}}{\lambda_{N I R}+\lambda_{S W I R}}
$$

\section{G. Assessment of maize foliar water status with spectral indices}

The combined RF and partial least square (PLS) were used for the predictive classification of the water status of the maize leaves. The RF+PLS algorithm was introduced by Boulesteix et al. (2008) and has the pre-validation idea that is based on cross-validation to avoid over-fitting embedded within the data set. It is fast and flexible in performing analysis of such huge data sets. The PLS has been found to produce satisfactory predictions (Zhang et al., 2012).

The bootstrap option of the RF+PLS used splits the data set of the indices developed. A total of 23 indices developed from the selected wavelengths (independent variables) and a sample size of $20 * 3$ water classes or status (total of 60 samples) were utilized for this algorithm. The training data set is used to construct and predict with the algorithm while the test is used to confirm predicted values into the various classes. The Overall accuracy and individual producer's as well as user's accuracies are later summarized for the 100 iterations performed per $n$ tree. For data sets having $\mathrm{n} \leq 30$, the minsplit parameter which controls the minimal size of nodes to be split within the bootstrap of the algorithm was defined as 4 (Boulesteix et al., 2008). 
The OOB is used to get a running adjusted unbiased estimate of the classification error as trees are added to the forest. This was applied in the RF+PLS algorithm to get estimates of index importance in the classification of the water status of maize leaves. In other words the OOB is obtained by comparing how much the error estimate increases when a variable is permuted while all other variables are left unchanged (Archer \& Kimes, 2008). For the RF+PLS, the ntree were tested at 50s up to 500 with an mtry of 5.

\section{Results and discussion}

The reflectance spectra of water stressed plants absorb less light in the visible and NIR regions of the spectrum than plants not experiencing water stress (Figure 3). The use of the leaf clip minimised atmospheric interferences, hence minimal cleaning of noise. The sample number for each category of water status measured had to be equalised at 20 which was the minimal number of samples per category, thereby establishing the total number of samples for this study as 60 .

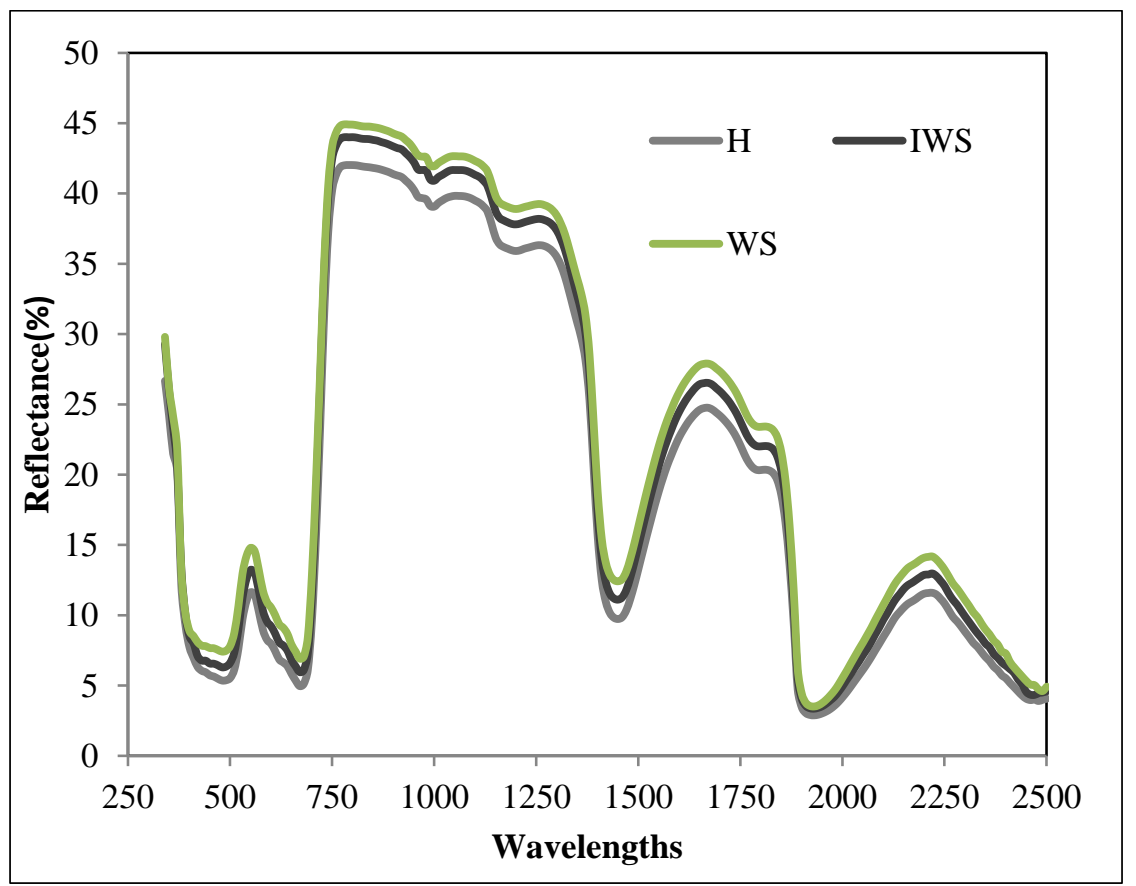

Figure 3: Leaf spectra for 3 categories of water status in maize leaves ( $\mathrm{H}$ is healthy or unstressed, IWS is intermediary stressed and WS is water stressed)

The spectra for the water stressed and unstressed maize leaves showed a distinctive difference on the visible, NIR and SWIR regions of the spectrum (Figure 4). The water stressed spectra recorded more reflectance in the visible region than unstressed ones because of the reduced chlorophyll content resulting from water deficiency that accounts for most of the absorption in this region. The alteration 
in the leaf structure also accounts for the visualised difference around the NIR region. This is as a result of the deficiency in liquid water within the plant tissues as explained by Canny and Huang (2006).

The combined algorithm of RF+PLS for the water indices produced an overall accuracy of $70 \pm 1.2 \%$ in distinguishing the different water stress levels for maize leaves under field conditions. The individual water stress categories obtained good producer's and user's accuracies (PA and UA) as follows: healthy, intermediary water stressed and water stressed of $63 \%, 94 \%$ and $65 \%$ for PA, and $73 \%, 76 \%$ and $71 \%$ for UA respectively.

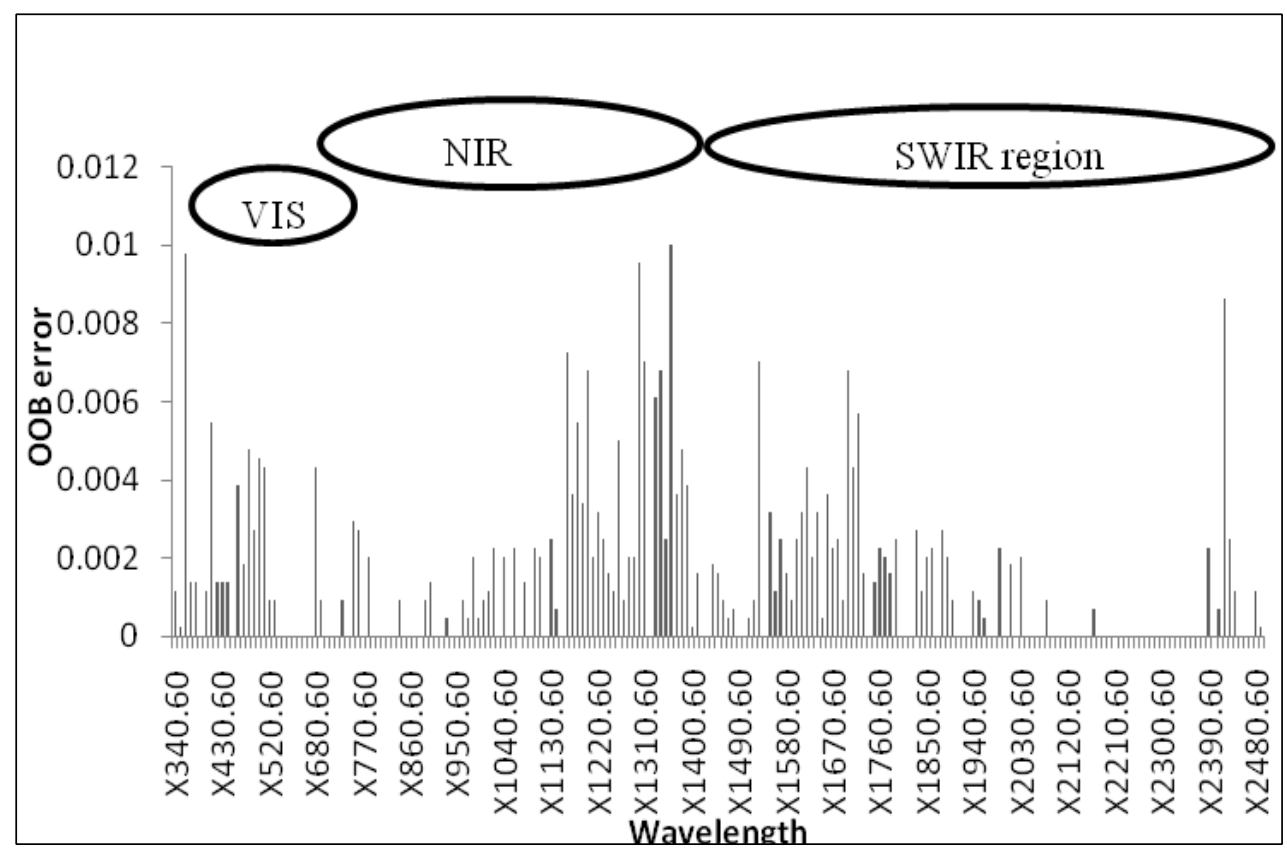

Figure 4: Wavelength selection for maize leaf water status and regions of the electromagnetic spectrum

Previous studies realized only the NIR and SWIR regions to be important in leaf water content assessment (Ceccato et al., 2001; Ceccato et al., 2002). In some recent studies the VIS region was also identified as important in water status assessment (Graeff \& Claupein, 2007; Genc et al., 2013). The detection of leaf water content in the VIS/NIR regions linking it to chlorophyll and nitrogen status was recently assessed and approved (Zhang et al., 2012). In another more recent study to establish wavelengths for the differentiation of various water levels in maize plants, the reflectance in the red region decreased and there was an increase in the NIR region. It established therefore that water stress could be detected by looking at the difference between the higher reflectance values obtained at the red region in comparison to the lower values at the NIR when the plant is not water 
stressed (Genc et al., 2013). The study reaffirmed the importance of the VIS region in water status detection in plants. Therefore, water stress in crops has the potential of disturbing the proper functioning of the process of photosynthesis.

The red edge region has also proven important in the detection of plant water content using spectral reflectance. For instance Liu et al. (2004) realized that the relationship between some of the known indices (WBI950/970 and NDWI860/1240) and the leaf water content for wheat was actually less significant but highly significant when the red edge position $(680-740 \mathrm{~nm})$ was compared with it. Graeff and Claupein (2007) discovered wavelength ratios of 510/780 nm and 540/780 nm as suitable in detecting water content in wheat grown under controlled conditions. Winterhalter et al. (2011) also identified the spectral indices from the VIS region ( $\mathrm{R}_{440} / \mathrm{R}_{685} ; \mathrm{R}_{525} / \mathrm{R}_{685} ; \mathrm{R}_{600} / \mathrm{R}_{680}$ and $\left.\mathrm{R}_{630} / \mathrm{R}_{680}\right)$ as highly significant in assessing relative water content in maize at pot levels and while at canopy level the significant index was the NDWI840/1650.

The variable of importance ranked the water-based indices according to their contribution in distinguishing maize water leaf status through a measure of their OOB error. The results obtained indicated the NDWI $860-1240$ as an important index for the monitoring of water stress in maize plants under field conditions (Figure 5). Other indices of importance included (1700-1530; 1530-1360). The WBI $950-970$ did not perform well in the monitoring of water stress levels in maize leaves under field conditions as proven by the variable importance results.

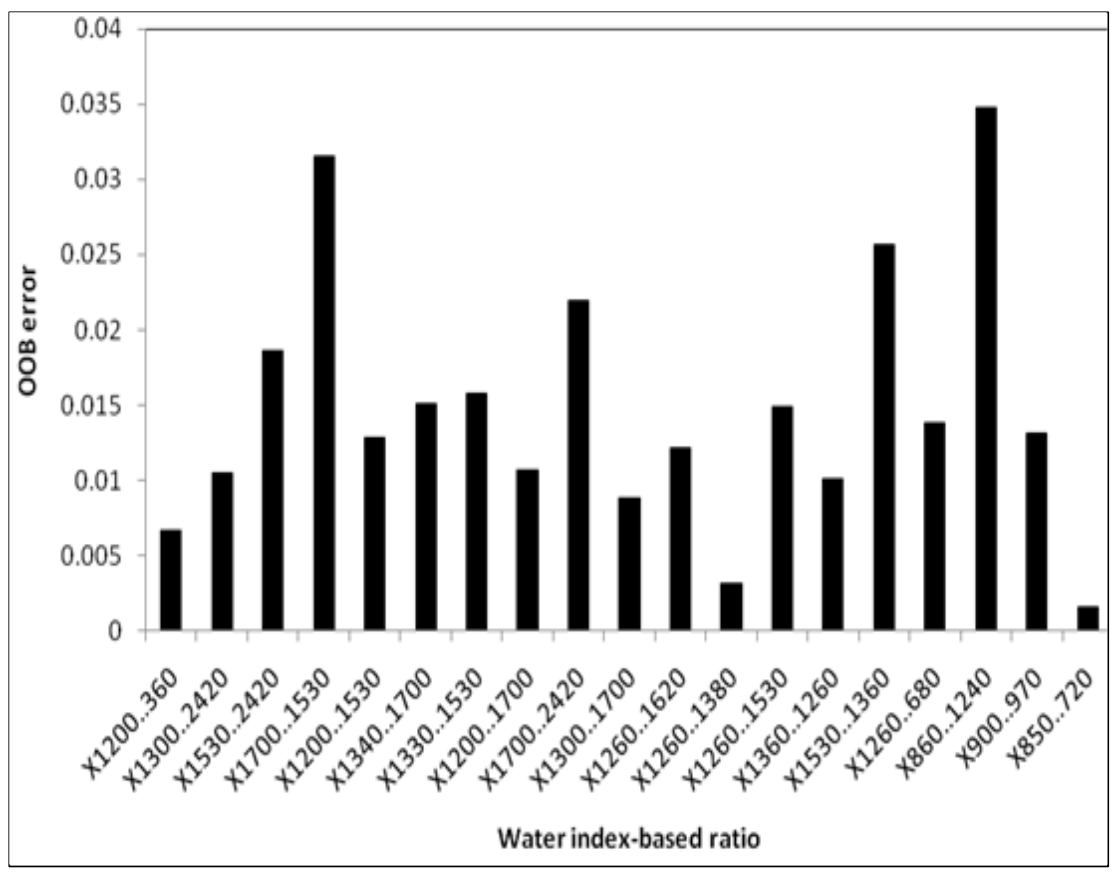

Figure 5: Selected indices for water stress level detection in maize 


\section{Conclusion}

The wavelengths of importance extracted as relevant in the assessment of maize leaf water status were actually located across the entire electromagnetic spectrum with a majority of them around the NIR and SWIR transition area (1300 and $1700 \mathrm{~nm})$. The red edge region did not show strong importance in the assessment of leaf water content of maize plants under field conditions. The few wavelengths in the VIS could be accounted for by the influence plant water status has on the chlorophyll concentration. While the minor and major water absorption regions around the NIR and SWIR showed stronger importance.

The developed ratios from the selected wavelengths in combination to the known water indices (WBI950-970 and NDWI860-1240) showed a successful prediction and classification of the leaf water status in the maize leaves under field conditions. The RF+PLS algorithm resulted in an OA of $70 \pm 1.2 \%$. The water-based ratios of importance extracted included the NDWI $(860-1240 ; 1700-1530 ; 1530-$ 1360). Therefore, spectroscopy could be used to assess the leaf water status of maize plants grown under field conditions.

Further research is required to be able to predict specific quantities of water in the leaf through well structured experiments. These experiments would control water applications to the plants and aid with the calculation of relative water content in the maize leaves through laboratory measurements that are required as ground-truth data.

\section{Acknowledgement}

We appreciate the University of Johannesburg-Common wealth scholarship for funding the studies and field support from the Department of Agriculture, Free State, South Africa. The input from the anonymous reviewers is also appreciated.

\section{References}

Archer, K.J. and R.V. Kimes, 2008: Empirical characterisation of random forest variable importance measures. Computational Statistics and Data Analysis 52, 2249-2260.

Boulesteix, A.L., C. Porzelius and M. Daumer, 2008: Microarray-based classification and clinical predictors: On combined classifiers and additional predictive value. Bioinformatics 24(15), 16981706. 10.1093/bioinformatics/btn262.

Breiman, L., 2001: Random Forests. Machine Learning 45(1), 5-32. 
Canny, M.J. and C.X. Huang, 2006: Leaf water content and palisade cell size. New Phytologist. 170(1), 75-85. DOI: 10.1111/j.1469-8137.2005.01633.x

Ceccato, P., S. Flasse, S. Tarantola, S. Jacquemoud and J-M Gregoire, 2001: Detection vegetation leaf water content using reflectance in the optical domain. Remote Sensing of Environment77, 22-33.

Ceccato, P., N. Gobron, S. Flasse, B. Pint and S. Tarantola, 2002: Designing a spectral index to estimate vegetation water content from remote sensing data: part 1: theoretical approach. Remote Sensing of Environment 82(2-3), 188-197.

Champagne, C.M., K. Staenz, A., Bannari, H. Mcnairin and J.C. Deguise, 2003: Validation of a hyperspectral curve fitting model for the estimation of plant water content of agricultural canopies. Remote Sensing of Environment 87,148-160.

Cleary, B. and J. Zaerr, 1984: Guidelines for measuring plant moisture stress with a pressure chamber. PMS Instrument Company. Royal Oaks Drive, Corvallis, Oregon, USA.

Clevers, J.G.P.W., L. Kooistra and M.E. Schaepman, 2008: Using spectral information from the NIR water absorption feature for the retrieval of canopy water content. International Journal of Applied Earth Observation and Geoinformation 10, 388-397.

Clevers, J.G.P.W., L. Kooistra and M.E. Schaepman, 2010: Estimating canopy water content using hyperspectral remote sensing data. International Journal of Applied Earth Observation and Geoinformation 12, 119-125.

Danson, F.M., M.D. Steven, T.J. Malthus and J.A. Clark, 1992: High-spectral resolution data for determining leaf water content. International Journal of Remote Sensing 13(3), 461-470.

de Jong, S.M., E.A. Addink and J.C. Doelman, 2014: Detecting leaf-water content in Mediterranean trees using high-resolution spectrometry. International Journal of Applied Earth Observation and Geoinformation 27, 128-136.

de Jong, S.M., E.A. Addink, P. Hoogenboom and W. Nijland, 2012: The spectral response of Buxussempervirens to different types of environmental stress, a laboratory experiment. ISPRS Journal of Photogrammetry and Remote Sensing 74, 56-65.

Eitel J.U.H., P.E. Gessler, A.M.S. Smith and R.Robberecht, 2006: Suitability of existing and novel spectral indices to remotely detect water stress in Populus spp. Forest Ecology and Management $229,170-182$. 
Elsayed, S., B. Misteleand U. Schmidhalter, 2011: Can changes in leaf water potential be assessed spectrally? Functional Plant Biology 38(6), 523-533.

Gao, B.C. 1996: NDWI-A normalized difference water index for remote sensing of vegetation liquid water from space.Remote sensing of Environment 58(3), 257-266.

Gaussman H.W., 1977: Reflectance of leaf components. Remote Sensing Environment 6,1-9.

Genc, L., M. Inalpulat, U. Kizil, M. Mirik, S. E. Smith and M. Mendes, 2013: Determination of water stress with spectral reflectance on sweet corn (Zea mays L.) using classification tree (CT) analysis. Zemdirbyste-Agriculture 100(1), 81-90.

Govender, M., P.J. Dye, I.M.Weiersbye, E.T.F.Witkowski and F Ahmed, 2009: Review of commonly used remote sensing and ground-based technologies to measure plant water stress, ISSN 0378-4738 (Print) = Water SA 35(5) ISSN 1816-7950 (On-line), 741-752.

Graeff, S. and W. Claupein, 2007: Identification and discrimination of water stress in wheat leaves (Triticumaestivum L.) by means of reflectance measurements. Irrigation Science 26(1), 61-70.

Hunt, E. R. and B. N. Rock, 1989: Detection of changes in leaf water content using near and middle-infrared reflectance. Remote Sensing of Environment 30, 43-54.

IPCC, 2007: IPCC Fourth Assessment Report: Climate Change 2007. Cambridge University Press, Cambridge.

Jackson T.J., D. Chen, M. Cosh, F. Li, M. Anderson, C. Walt-Hall, P. Doriaswamy and E.R. Hunt, 2004: Vegetation water content mapping using Landsat data derived normalized difference water index for corn and soybeans. Remote Sensing of Environment 92, 475-482.

Liu, L., J. Wang, W. Huang, C. Zhao, B. Zhang and Q. Tong, 2004: Estimating winter wheat plant water content using red edge parameters. International Journal of Remote Sensing 17, 33313342.

Maracci, G., G. Schmuck, B. Hosgood and G. Andreoli, 1991: Interpretation of reflectance spectra by plant physiological parameters. In: Proceedings of International Geoscience and Remote Sensing Symposium (IGARSS'91), Remote Sensing: Global Monitoring for Earth Management. Helsinki University of Technology, Espoo (Finland), 3 - 6 June 1991 (pp. 2303 - 2306). New York: IEEE.

Peñuelas, J., I. Filella, C. Biel, L. Serrano and R. Save, 1993: The reflectance at the 950-970 region as an indicator of plant water status. International Journal of Remote Sensing 14(10), 1887-1905. 
Peñuelas, J., J. Piñol, R. Ogaya, and I. Filella, 1997: Estimation of plant water concentration by the reflectance Water Index WI (R900/R970). International Journal of Remote Sensing 18(13), 2869-2875.

PSR Series Spectroradiometer Manual Revision: 1.05. 9/24/2012

RStudio 2015: RStudio: Integrated development environment for R (Version 0.96.122) [Computer software]. Boston, MA. Retrieved January 20, 2015. Available from http://www.rstudio.org/

Sims, D.A. and J.A. Gamon, 2003: Estimation of vegetation water content and photosynthetic tissue area from spectral reflectance: a comparison of indices based on liquid water and chlorophyll absorption features. Remote Sensing of Environment 84(4), 526-537.

Strobl, C., A-L. Boulesteix, T. Kneib, T. Augustin and A. Zeileis, 2008: Conditional variable importance for random forests. BMC Bioinformatics, available online at: http://www.biomedcentral.com/1471-2105/9/307 Accessed 30/03/2015.

Strobl, C., Boulesteix, A., A. Zeileis and T. Hothorn, 2007: Bias in random forest variable importance measures: illustrations, sources and a solution. BMC Bioinformatics, 8: 1-21.

Suo, X.M., Y.T. Jiang, M. Yang, S.K. Li, K.R. Wang and C.T. Wang, 2010: Artificial neural network to predict leaf population chlorophyll content from cotton plant images. Agricultural Sciences in China 9(1), 38-45.

Tucker, C.J., 1980: Remote sensing of leaf water content in the near infrared. Remote Sensing of Environment 10, 23-32.

Winterhalter, L, B, Mistele, S, Jampatong and U. Schmidhalter, 2011: High throughput sensing of aerial biomass and above-ground nitrogen uptake in the vegetative stage of well-watered and drought stressed tropical maize hybrids. Crop Science 51, 479-489. doi:10.2135/cropsci2010.07.0397

Zhang, Q., Q. Li, and G. Zhang, 2012: Rapid determination of leaf water content using VIS/NIR spectroscopy analysis with wavelength selection. Spectroscopy: An International Journal 27(2), 93-105. 